\title{
Open Mathematics
}

\section{Research Article}

\section{Primitivo B. Acosta-Humánez*, Alberto Reyes-Linero, and Jorge Rodriguez-Contreras Galoisian and qualitative approaches to linear Polyanin-Zaitsev vector fields}

https://doi.org/10.1515/math-2018-0102

Received March 7, 2018; accepted June 13, 2018.

\begin{abstract}
In this paper we study a particular parametric family of differential equations, the so-called Linear Polyanin-Zaitsev Vector Field, which has been introduced in a general case in [1] as a correction of a family presented in [2]. Linear Polyanin-Zaitsev Vector Field is transformed into a Liénard equation and, in particular, we obtain the Van Der Pol equation. We present some algebraic and qualitative results to illustrate some interactions between algebra and the qualitative theory of differential equations in this parametric family.
\end{abstract}

Keywords: Integrability, Qualitative, Stability, Critical point, Polynomial, Polyanin-Zaitsev vector field

MSC: 12H05, 34C99

\section{Introduction}

The analysis of dynamical systems has been a topic of great interest for many mathematicians and theoretical physicists since the seminal works of $\mathrm{H}$. Poincaré. Every system is dynamical when it is changing with time. It was H. Poincare who introduced the qualitative approach to study dynamical systems, while E. Picard and E. Vessiot introduced an algebraic approach to study linear differential equations based on the Galois theory for polynomials, see [3].

An important family of dynamical systems are Van der Pol type systems. The forced Van der Pol chaotic oscillator was discovered by Van der Pol and Van der Mark ([4], 1927). The Van der Pol oscillator has a long history of being used in both physical and biological sciences. For instance, in biology Fitzhugh [5] and Nagumo [6] extended the Van der Pol equation in a planar field as a model for action potentials of neurons. A detailed study on forced Van der Pol equation is found in [7].

The Handbook of Nonlinear Partial Differential Equations [2], a unique reference for scientists and engineers, contains over 3,000 nonlinear partial differential equations with solutions, as well as exact, symbolic, and numerical methods for solving nonlinear equations. First-, second-, third-, fourth-, and higherorder nonlinear equations and systems of equations are considered. A writing errata presented in one of these problems was corrected in [1]. This problem in correct form was used and studied in [8]. The differential equation system associated to this problem called "Polyanin-Zaitsev vector field" [1], has as associated foliation a Lienard equation, that is to say, it is closely related to a problem of the Van Der Pol type.

\footnotetext{
^Corresponding Author: Primitivo B. Acosta-Humánez: Universidad del Simón Bolívar, Barranquilla, Colombia \& Instituto Superior de Formación Docente Salomé Ureña - ISFODOSU, Recinto Emilio Prud'Homme, Santiago de los Caballeros, Dominican Republic, E-mail: primitivo.acosta@unisimonbolivar.edu.co

Alberto Reyes-Linero: Universidad del Atlántico, Barranquilla, Colombia, E-mail: areyeslinero@mail.uniatlantico.edu.co Jorge Rodriguez-Contreras: Universidad del Norte \& Universidad del Atlántico, Barranquilla, Colombia, E-mail: jrodri@uninorte.edu.co
} 
In this paper we study one parametric family of linear differential systems from algebraic and qualitative point of view. Such parametric family comes from the correction of Exercise 11 in [2, §1.3.3], which we called Polyanin-Zaitsev vector field, see [1]. We find the critical points and we describe the orbits behavior in near of this points. In the algebraic aspects, we obtain the explicit first integral through Darboux method. Moreover, we compute the differential Galois group associated to such systems.

\section{Preliminaries}

A polynomial system in the plane of degree $n$ is given by

$$
\begin{aligned}
& \dot{x}=P(x, y) \\
& \dot{y}=Q(x, y),
\end{aligned}
$$

where $P, Q \in \mathbb{C}[x, y]$ (set of polynomials in two variables) and $n$ is the absolute degree of the polynomials $P$ and $Q$.

The polynomial vector field associated with the system (1) is given by $X:=(P, Q)$, which can also be written as:

$$
\mathrm{X}=P(x, y) \frac{\partial}{\partial x}+Q(x, y) \frac{\partial}{\partial y} .
$$

A foliation of a polynomial vector field of the form (1) is given by

$$
\frac{d y}{d x}=\frac{Q(x, y)}{P(x, y)} \text {. }
$$

Given the family of equations

$$
y y^{\prime}=\left(a(2 m+k) x^{m+k-1}+b(2 m-k) x^{m-k-1}\right) y-\left(a^{2} m x^{4 k}+c x^{2 k}+b^{2} m\right) x^{2 m-2 k-1} .
$$

Then the system of associated equations has the form:

$$
\begin{aligned}
& \dot{x}=y \\
& \dot{y}=\left(a(2 m+k) x^{m+k-1}+b(2 m-k) x^{m-k-1}\right) y-\left(a^{2} m x^{4 k}+c x^{2 k}+b^{2} m\right) x^{2 m-2 k-1},
\end{aligned}
$$

see [1].

We can see two important theorems for the study of infinity behavior: the theorem 1 (see [9, §3.10-P 271]), which allows us to find the infinity critical points and the theorem 2 (see [9, §3.10-P 272, 273]), which allows us tu characterize these points.

The following theorem is also of vital importance for our study and can be seen in greater detail in [12] and [13].

Theorem 1.1 (Darboux). Suppose that the polynomial system (1) of degree $m$ admits $p$ irreducible algebraic invariant curves $f_{i}=0$ with cofactors $K_{i}$ for $i=1, \ldots, p$; $q$ exponential factor $F_{j}=\exp \left(g_{j} / h_{j}\right)$ with cofactors $L_{j}$ for $j=1, \ldots, q$ and $r$ independents single points $\left(x_{k}, y_{k}\right) \in \mathbb{C}^{2}$ such that $f_{i}\left(x_{k}, y_{k}\right) \neq 0$ for $i=1, \ldots, p$ and $k=1, \ldots, p$, also $h_{j}$ factor in factor product $f_{1}, f_{2}, \ldots, f_{q}$ except if it is equal to 1 . So the following statements are kept:

a) There exists $\lambda_{i}, \mu_{i} \in \mathbb{C}$ not all null, such that

$$
\sum_{i=1}^{p} \lambda_{i} K_{i}+\sum_{j=1}^{q} \mu_{j} L_{j}=0, \quad\left(D_{f i}\right)
$$

if and only if the function (multi-valued)

$$
H(x, y)=f_{1}^{\lambda_{1}} \ldots f_{p}^{\lambda_{p}} F_{1}^{\mu_{1}} \ldots F_{q}^{\mu_{q}},
$$


is a first integral of the system (1). Moreover, when is (1) is a real systems, $(H)$ is a real function.

b) If $p+q+r=[m(m+1) / 2]+1$ then there exists $\lambda_{i}, \mu_{i} \in \mathbb{C}$ not all zero such that they satisfy the condition $\left(D_{f i}\right)$.

c) If $p+q+r=[m(m+1) / 2]+2$ then the system (1) has a rational first integral and all orbits of the system are contained in some invariant algebraic curve.

d) There exists $\lambda_{i}, \mu_{i} \in \mathbb{C}$ not all zero such that

$$
\sum_{i=1}^{p} \lambda_{i} K_{i}+\sum_{j=1}^{q} \mu_{j} L_{j}+\operatorname{div}(P, Q)=0, \quad\left(D_{i f}\right)
$$

if and only if the function (2) is a first integral of the system (1). Moreover, for the real systems the function (2) is real.

e)If $p+q+r=m(m+1) / 2$ and the $r$ singular points are independents, then exist $\lambda_{i}, \mu_{i} \in \mathbb{C}$ not all zero such that they satisfy any of the conditions $\left(D_{f}\right) o\left(D_{i f}\right)$.

f) There exist $\lambda_{i}, \mu_{i} \in \mathbb{C}$ not all zero such that

$$
\sum_{i=1}^{p} \lambda_{i} K_{i}+\sum_{j=1}^{q} \mu_{j} L_{j}+s=0, \quad\left(D_{i n}\right)
$$

with $s \in \mathbb{C}-\{0\}$ if and only if the function (multi-valued)

$$
I(x, y, t)=f_{1}^{\lambda_{1}} \ldots f_{p}^{\lambda_{p}} F_{1}^{\mu_{1}} \ldots F_{q}^{\mu_{q}} \exp (s t),
$$

is an invariant of the system (1). Moreover, for the real systems function (3) is real.

Now we write the Polyanin-Zaitsev vector field, introduced in [1]:

$$
X:=y \frac{\partial}{\partial x}+\left(\left(\alpha x^{m+k-1}+\beta x^{m-k-1}\right) y-\gamma x^{2 m-2 k-1}\right) \frac{\partial}{\partial x},
$$

where $\alpha=a(2 m+k), \beta=b(2 m-k)$ y $\gamma(x)=a m x^{4 k}+c x^{2 k}+b m$.

The differential system associated with the Polyanin-Zaitsev vector field (2) is:

$$
\begin{aligned}
& \dot{x}=y \\
& \dot{y}=\left(\alpha x^{m+k-1}+\beta x^{m-k-1}\right) y-\gamma x^{2 m-2 k-1} .
\end{aligned}
$$

The aim of this paper is to analyze, from Galoisian and qualitative point of view, the complete set of families where the Polyanin-Zaitsev differential system (3) is a linear differential system.

\section{Conditions for the problem}

The following lemma allows us to identify the linear cases associated with the Polyanin-Zaitsev vector field.

Lemma 2.1. The Polyanin-Zaitsev differential system is a linear system, with $Q$ not null polynomial, if is equivalently affine to one of the following families:

$$
\begin{aligned}
& \dot{x}=y \\
& \dot{y}=-c x
\end{aligned}
$$

With critical point $(0,0)$, saddle node.

$$
\begin{aligned}
& \dot{x}=y \\
& \dot{y}=b(k+2) y-b^{2}(k+1) x .
\end{aligned}
$$

With critical point $(0,0)$.

$$
\begin{aligned}
& \dot{x}=y \\
& \dot{y}=a(2-k) y-(1-k) a^{2} x
\end{aligned}
$$


With critical point $(0,0)$.

$$
\begin{aligned}
& \dot{x}=y \\
& \dot{y}=2 b y-b^{2} x-c x .
\end{aligned}
$$

With critical point $(0,0)$.

$$
\begin{aligned}
& \dot{x}=y \\
& \dot{y}=\frac{3}{2} b y-\frac{b^{2}}{2} x-c .
\end{aligned}
$$

With critical point of the form $\left(\frac{-2 c}{b^{2}}, 0\right)$.

$$
\begin{aligned}
& \dot{x}=y \\
& \dot{y}=2 a y-a^{2} x-c x .
\end{aligned}
$$

The critical point is $(0,0)$.

$$
\begin{aligned}
& \dot{x}=y \\
& \dot{y}=\frac{3}{2} a y-\frac{a^{2}}{2} x-c .
\end{aligned}
$$

The critical point are of the form $\left(\frac{-2 c}{a^{2}}, 0\right)$.

$$
\begin{aligned}
& \dot{x}=y \\
& \dot{y}=2(a+b) y-\left(a^{2}+b^{2}\right) x
\end{aligned}
$$

With critical point $(0,0)$.

$$
\begin{aligned}
& \dot{x}=y \\
& \dot{y}=2(a+b) y-\left(a^{2}+c+b^{2}\right) x
\end{aligned}
$$

With critical point $(0,0)$.

Proof. We will analyze every possible cases for the constants $a, b$ y $c$.

Case 1:If $a=b=0$ and $c \neq 0$, the system (3) is reduced to:

$$
\begin{aligned}
& \dot{x}=y \\
& \dot{y}=-c x^{2 m-1} .
\end{aligned}
$$

Given the conditions of the problem $2 m-1=1$, the associated system is

$$
\begin{aligned}
& \dot{x}=y \\
& \dot{y}=-c x .
\end{aligned}
$$

Case 2:If $a=c=0$ and $b \neq 0$. In this case the system (3) is reduced to:

$$
\begin{aligned}
& \dot{x}=y \\
& \dot{y}=b(2 m-k) y x^{m-k-1}-b^{2} m x^{2 m-2 k-1} .
\end{aligned}
$$

It system is linear if $m-k-1=0$ that is $2 m-2 k-1=1,2 m-k=k+2$ and $m=k+1$, then the associated system is:

$$
\begin{aligned}
& \dot{x}=y \\
& \dot{y}=b(k+2) y-b^{2}(k+1) x .
\end{aligned}
$$

Case 3:If $b=c=0$ and $a \neq 0$. In this case the system (3) is reduced to:

$$
\begin{aligned}
& \dot{x}=y \\
& \dot{y}=a(2 m+k) y x^{m+k-1}-a^{2} m x^{2 m+2 k-1} .
\end{aligned}
$$

The system is linear if $m+k-1=0$ that is $2 m+2 k-1=1,2 m+k=2-k$ and $m=1-k$, then the associated system is

$$
\begin{aligned}
& \dot{x}=y \\
& \dot{y}=a(2-k) y-a^{2}(1-k) x .
\end{aligned}
$$


Case 4:If $a=0, b \neq 0$ and $c \neq 0$, the system (3) is reduced to:

$$
\begin{aligned}
& \dot{x}=y \\
& \dot{y}=b(2 m-k) y x^{m-k-1}-b^{2} m x^{2 m-2 k-1}-c x^{2 m-1} .
\end{aligned}
$$

$m-k-1=0$ then $2 m-2 k-1=1$, but in this case we have two options $2 m-1=0$ or $2 m-1=0$, that is to, the two linear system are:

$$
\begin{aligned}
& \dot{x}=y \\
& \dot{y}=2 b y-b^{2} x-c x .
\end{aligned}
$$

and

$$
\begin{aligned}
& \dot{x}=y \\
& \dot{y}=\frac{3}{2} b y-\frac{b^{2}}{2} x-c .
\end{aligned}
$$

Case 5:If $b=0, a \neq 0$ and $c \neq 0$, the system (3) is reduced to:

$$
\begin{aligned}
& \dot{x}=y \\
& \dot{y}=a(2 m+k) y x^{m+k-1}-a^{2} m x^{2 m+2 k-1}-c x^{2 m-1} .
\end{aligned}
$$

$m+k-1=0$ then $2 m+2 k-1=1$, and we have two options $2 m-1=0$ or $2 m-1=0$, that is, the two linear system are:

$$
\begin{aligned}
& \dot{x}=y \\
& \dot{y}=2 a y-a^{2} x-c x .
\end{aligned}
$$

and

$$
\begin{aligned}
& \dot{x}=y \\
& \dot{y}=\frac{3}{2} a y-\frac{a^{2}}{2} x-c .
\end{aligned}
$$

Case 6: If $c=0, a \neq 0$ and $b \neq 0$, the system (3) is reduced to:

$$
\begin{aligned}
& \dot{x}=y \\
& \dot{y}=a(2 m+k) y x^{m+k-1}-a^{2} m x^{2 m+2 k-1}+b(2 m-k) y x^{m-k-1}-b^{2} m x^{2 m-2 k-1} .
\end{aligned}
$$

In this case $m+k-1=0$ and $m-k-1=0$, then $m=1$ and $k=0$. The asociated system is:

$$
\begin{aligned}
& \dot{x}=y \\
& \dot{y}=2(a+b) y-\left(a^{2}+b^{2}\right) x
\end{aligned}
$$

Case 7:c $\neq 0, a \neq 0$ and $b \neq 0$, then we have the system (3). Again we have that $m+k-1=0$ and $m-k-1=0$, and then the resulting system is

$$
\begin{aligned}
& \dot{x}=y \\
& \dot{y}=2(a+b) y-\left(a^{2}+c+b^{2}\right) x .
\end{aligned}
$$

A qualitative and Galoisian detailed study is carried out over the linear system (12) of the previous lemma, the rest of cases are studied in a similar form. 


\section{Critical points}

Proposition 3.1. The following statements hold:

i. If $c>0,(0,0)$ is the only critical point on the finite plane, it is also stable.

ii. If $a>0, b>0$ and $c>0$, the critical points at infinity for the system (12) are given by:

$$
(x, y, 0)=\left( \pm \sqrt{1-y^{2}},(a+b) \pm \sqrt{2 a b-c}, 0\right) .
$$

Proof. Let us analyze now for (12) the critical points in both the finite and infinity planes. For this the Theorem 1 (see [9, §3.10-P 271]) is used, which is based on the study of the system, on the equator of the Poincare sphere, that is when $x^{2}+y^{2}=1$.

Remember that $P(x, y)=y$ y $Q(x, y)=2(a+b) y-\left(a^{2}+b^{2}+c\right) x$ with $a, b, c>0$, then the critical points are given by the system:

$$
\begin{aligned}
y & =0 \\
2(a+b) y-\left(a^{2}+b^{2}+c\right) x & =0
\end{aligned}
$$

then the only critical point in the finite plane is $(x, y)=(0,0)$. Now for the qualitative analysis of the critical point we find the Jacobian matrix evaluated in the point, given by:

$$
\left.D(P, Q)\right|_{(0,0)}=\left(\begin{array}{cc}
0 & 1 \\
-\left(a^{2}+b^{2}+c\right) & 2(a+b) .
\end{array}\right)
$$

The characteristic polynomial is

$$
\lambda^{2}-2(a+b) \lambda+\left(a^{2}+b^{2}+c\right)=0,
$$

then the eigenvalues are:

$$
\lambda_{1,2}=\frac{2(a+b) \pm \sqrt{4(a+b)^{2}-4\left(a^{2}+b^{2}+c\right)}}{2}=(a+b) \pm \sqrt{2 a b-c} .
$$

According to Theorem 1 (see [9, §3.10-P 271]) and Theorem 2 (see [9, §3.10-P 272, 273]), to find the critical points and their behavior we can use the system:

$$
\begin{aligned}
& { }_{ \pm} \dot{y}=y z^{r} P(1 / z, y / z)-z^{r} Q(1 / z, y / z) \\
& { }_{ \pm} \dot{z}=z^{r+1} P(1 / z, y / z) .
\end{aligned}
$$

In our case $r=1$ and when replacing we find:

$$
\begin{aligned}
& \pm \dot{y}=y^{2}-2(a+b) y+\left(a^{2}+b^{2}+c\right) p \\
& \pm \dot{z}=z y
\end{aligned}
$$

The critical points for this system result from solving the system:

$$
\begin{aligned}
& y^{2}-2(a+b) y+\left(a^{2}+b^{2}+c\right)=0 \\
& z=0
\end{aligned}
$$

then

$$
y_{1,2}=\frac{2(a+b) \pm \sqrt{4(a+b)^{2}-4\left(a^{2}+b^{2}+c\right)}}{2}=(a+b) \pm \sqrt{2 a b-c} .
$$

Also, as we are in the equator of the Poincare sphere $x^{2}+y^{2}=1$ that is $x= \pm \sqrt{1-y^{2}}$.

We note that if we change the sings of $x$ e and we obtain points called Antipodes, which have a contrary stability. Taking into account the above conditions, the critical points at infinity are:

$$
(x, y, 0)=\left( \pm \sqrt{1-y^{2}},(a+b) \pm \sqrt{2 a b-c}, 0\right) .
$$


The Jacobian matrix (14) is:

$$
D(P, Q)=\left(\begin{array}{cc}
2 y-2(a+b) & 0 \\
z & 0
\end{array}\right)
$$

in our case:

$$
\left.D(P, Q)\right|_{\left(y_{1,2}, 0\right)}=\left(\begin{array}{cc} 
\pm 2 \sqrt{2 a b-c} & 0 \\
0 & (a+b) \pm \sqrt{2 a b-c} .
\end{array}\right)
$$

We can notice then that the signs of the own values depend on the signs of the elements in the diagonal. The following indicates that several cases must be analyzed for both the finite and infinity planes, be $\Delta=2 a b-c$.

Case 1. If $\Delta>0$ then in (13) $\lambda_{1}>0$, but for $\lambda_{2}$ we have three options. If $\lambda_{2}<0$ and $\lambda_{2}=0$, contradicts the fact that $a, b, c>0$ then the only option is $\lambda_{2}>0$. Since both eigenvalues are positive then the origin is a critical point repulsor.

Regarding the critical points at infinity in this case, we can see the elements on the diagonal of (14) have two options $y_{1}, y_{2}$. For $y_{1}$ :

$$
\left.D(P, Q)\right|_{\left(y_{1}, 0\right)}=\left(\begin{array}{cc}
2 \sqrt{2 a b-c} & 0 \\
0 & (a+b)+\sqrt{2 a b-c}
\end{array}\right)
$$

For this point, the values in the diagonal are positive and then in infinity this critical point is of the repulsor type and, therefore, its antipode is of an attractor type.

For $y_{2}$ :

$$
\left.D(P, Q)\right|_{\left(y_{2}, 0\right)}=\left(\begin{array}{cc}
-2 \sqrt{2 a b-c} & 0 \\
0 & (a+b)-\sqrt{2 a b-c}
\end{array}\right)
$$

where $-2 \sqrt{2 a b-c}<0$ and $(a+b)-\sqrt{2 a b-c}=\lambda_{2}>0$ Therefore, the critical point is of a saddle type. So the phase portrait for the system in this case is:

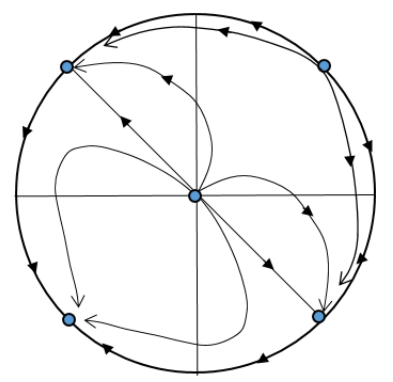

Global phase portrait for case 1 (12)

Case 2. If $\Delta=0$ then $\lambda_{1}=\lambda_{2}>0$ that is, the origin is a repulsor critical point.

For the points at infinity the system (14) is reduced to:

$$
\begin{aligned}
& \pm \dot{y}=(y-a-b)^{2} \\
& \pm \dot{z}=z y
\end{aligned}
$$

with solution $y=a+b>0$. Then the phase portrait for the system in this case is: 


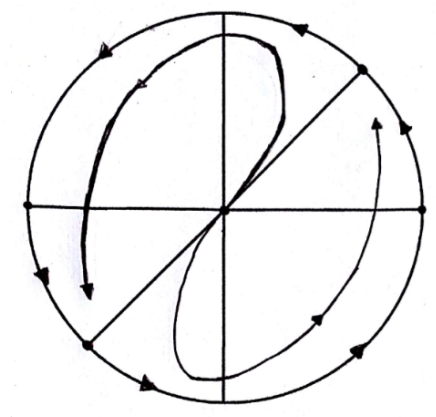

Global phase portrait for case 2 (12)

Case 3. If $\Delta<0$ then $\lambda_{1}=(a+b)+i \sqrt{c-2 a b}$ and $\lambda_{2}=(a+b)-i \sqrt{c-2 a b}$ with $(a+b)>0$ that is the origin is a critical point of a spiral type. So the phase portrait for the system in this case is:

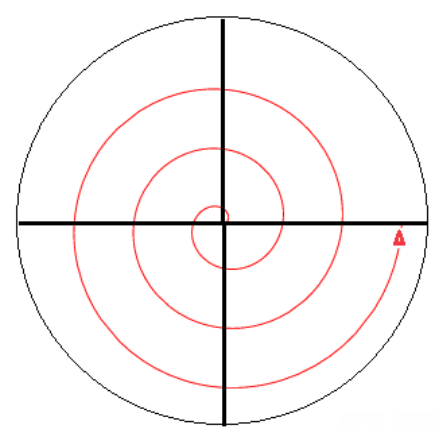

Global phase portrait for case 3 (12)

As regards infinity, the equation (15) has no real solutions, that is, it has no critical points at infinity.

\section{Bifurcations}

Lemma 4.1. Let $B_{0}:=\left\{(a, b, c): a^{2}+b^{2}<-c\right\}$. if $(a, b, c) \in B_{0}$ then the associated system of the form (12) has unstable critical point $(0,0)$.

Proof. We consider eigenvalues (13), if $(a, b, c) \in B_{0}$ that is $a^{2}+b^{2}<-c$, then $(a+b)^{2}<2 a b-c$, it is $0<(a+b)<\sqrt{2 a b-c}$.

I. If $a+b>0$, then $a+b<\sqrt{2 a b-c}$, i.e.

$$
\lambda_{2}=\frac{a+b-\sqrt{2 a b-c}}{2}<0 \quad \& \quad \lambda_{1}=\frac{a+b+\sqrt{2 a b-c}}{2}>0 .
$$

This implies that the critical point $(0,0)$ is of a saddle type.

II. If $a+b<0$, then $-(a+b)<\sqrt{2 a b-c}$, i.e.

$$
\lambda_{1}=\frac{a+b+\sqrt{2 a b-c}}{2}>0 \quad \& \quad \lambda_{2}=\frac{a+b-\sqrt{2 a b-c}}{2}<0 .
$$

Then the critical point $(0,0)$ is of a saddle type.

III. If $a+b=0$ then $\lambda_{1}=\sqrt{2 a b-c}>0$ and $\lambda_{2}=-\sqrt{2 a b-c}<0$. Then the critical point $(0,0)$ is of a sadle type. 
Lemma 4.2. Let $B_{1}:=\{(a, b, c): 2 a b-c<0 \& a+b>0\}$. if $(a, b, c) \in B_{1}$ then the associated system of the form (12) has unstable focus critical point $(0,0)$.

Proof. We consider the eigenvalues (13), if $(a, b, c) \in B_{1}$, then $0<(a+b)^{2}>0>2 a b-c$, i.e. that the eigenvalues are:

$$
\lambda_{1}=\frac{a+b+i \sqrt{c-2 a b}}{2}<0 \quad \& \quad \lambda_{2}=\frac{a+b-i \sqrt{c-2 a}}{2}<0 .
$$

where the real part in both cases is $(a+b)>0$, i.e. the critical point is focus stable.

Lemma 4.3. Let $B_{2}:=\{(a, b, c): 2 a b-c<0 \& a+b<0\}$. If $(a, b, c) \in B_{2}$ the associated system of the form (12) has a critical point of saddle type $(0,0)$.

Proof. The proof of this lemma is as in the previous lemma, but in this case $a+b<0$, then the critical point is focus stable.

Lemma 4.4. Let $B_{3}:=\left\{(a, b, c): 2 a b-c \geq 0 \& a+b>0 \& a^{2}+b^{2}>-c\right\}$. If $(a, b, c) \in B_{3}$ then the associated system of the form (12) has a critical point of unstable node type $(0,0)$.

Proof. We consider the eigenvalues (13), if $(a, b, c) \in B_{3}$, then $a^{2}+b^{2}>-c$, that is $(a+b)^{2}>2 a b-c>0$, i.e.

$$
\lambda_{1}=a+b+\sqrt{2 a b-c}>0 \quad \& \quad \lambda_{2}=a+b-\sqrt{2 a b-c}>0 .
$$

Then the critical point $(0,0)$ is an unstable node.

Lemma 4.5. Let $B_{4}:=\left\{(a, b, c): 2 a b-c \geq 0 \& a+b<0 \& a^{2}+b^{2}>-c\right\}$. If $(a, b, c) \in B_{4}$ then the associated system of the form (12) has a critical point stable node type $(0,0)$.

Proof. If $(a, b, c) \in B_{4}$, then $|a+b| \geq \sqrt{2 a b-c}$, but $a+b<0$ that is $-(a+b) \geq \sqrt{2 a b-c}$. Now the eigenvalues are:

$$
\lambda_{1}=a+b+\sqrt{2 a b-c}<0 \quad \& \quad \lambda_{2}=a+b-\sqrt{2 a b-c}<0 .
$$

Then the critical point $(0,0)$ is a stable node.

Proposition 4.6. The set $B:=\left\{(a, b, c): a+b<0 \& a^{2}+b^{2}=-c\right\} \cup\left\{(a, b, c): a+b=0 \& a^{2}+b^{2}>-c\right\}$ is a bifurcation for the system (12).

Proof. For the previous lemmas, the set $B$ is a bifurcation for the family of systems (12).

Remark 4.7. The Proposition (4.6) us present a new kind of bifurcation.

The following corollary summarizes the study of the bifurcations for the rest of families and is a direct consequence of the proposition (4.6).

Corollary 4.8. The following statements hold:

1. For the family (4), $c=0$ is a bifurcation. For the family (5) there is no bifurcation. For the family (6) $a=0$ is a bifurcation.

2. For the families (7) and (8), the set $F_{1}:=\left\{(b, c): b=0 \& b^{2}>-c\right.$ or $\left.b^{2}=-c \& b>0\right\}$ is $a$ bifurcation.

3. For the families (9) and (10), the set $F_{2}:=\left\{(a, c): a=0 \& a^{2}>-c\right.$ or $\left.a^{2}=-c \& a>0\right\}$ is $a$ bifurcation.

4. For the family (11) the set $F_{3}:=\{(a, b): a+b=0\}$ is a bifurcation. 


\section{Galoisian aspects}

To simplify the next proposition, we summarize all the possibilities for parameter $\rho$ in Table 1 .

Table 1. Possibles $\rho$ values.

\begin{tabular}{|c|c|c|c|c|}
\hline$a$ & $\boldsymbol{b}$ & c & $\rho$ & Differential Galois Group \\
\hline 0 & 0 & $\neq 0$ & $-c$ & $\mathbb{G}_{m}$ \\
\hline 0 & $\neq \mathbf{0}$ & 0 & 0 & $\mathbb{G}_{a}$ \\
\hline$\neq 0$ & 0 & 0 & 0 & $\mathbb{G}_{a}$ \\
\hline$\neq 0$ & $\neq \mathbf{0}$ & $\mathbf{0}$ & $2 a b$ & $\mathbb{G}_{m}$ \\
\hline$\neq 0$ & $\mathbf{0}$ & $\neq 0$ & $-c$ & $\mathbb{G}_{m}$ \\
\hline 0 & $\neq \mathbf{0}$ & $\neq 0$ & $-c$ & $\mathbb{G}_{m}$ \\
\hline$\neq 0$ & $\neq \mathbf{0}$ & $\neq 0$ & $2 a b-c$ & $\mathbb{G}_{m}$ \\
\hline
\end{tabular}

Proposition 5.1. For (12), with $\rho=2 a b-c$ we have that:

i. If $(2 a b-c)=0$ then the differential Galois group is

$$
D G a l<L / K>=\left(\left\{\sigma_{c}: \sigma_{c}(y)=y+c \quad c \in \mathbf{C}\right\}, o\right)
$$

ii. If $(2 a b-c) \neq 0$ then the differential Galois group is

$D G a l<L / K>=\left(\left\{\sigma_{c} / \sigma_{c}(y)=c y \quad c \in \mathbf{C}\right\}, o\right)$

Proof. The associated foliation to (12) is:

$$
y y^{\prime}=(2 a+2 b) y-\left(a^{2}+c+b^{2}\right) x .
$$

Then taking into account that $f(x)=-2(a+b) y$ and $g(x)=\left(a^{2}+c+b^{2}\right) x$, the Lienard equation will be:

$$
\ddot{x}-(2 a+2 b) \dot{x}+\left(a^{2}+c+b^{2}\right) x=0 .
$$

This is a second order equation with constant coefficients.

If we take it to the form $\ddot{y}=\rho y$ with the change of variable $x=\exp \left(-1 / 2 \int f(x) d t\right)$ that is to say $x=$ $\exp ((a+b) t) y$, we obtain:

$$
\ddot{y}=(2 a b-c) y .
$$

Let's analyze each case of the solution of the associated Lienard equation taking into account the following: $(2 a b-c)=\rho$. The solution of the second order reduced equation is $y(t)=C_{1} \exp (\sqrt{\rho} t)+C_{2} \exp (-\sqrt{\rho} t)$ if they are taken $\left(C_{1}, C_{2}\right)=(1,0)$ or $\left(C_{1}, C_{2}\right)=(0,1)$ and the particular solutions are

$$
y_{1}=\exp (\sqrt{\rho} t) \quad y_{2}=\exp (-\sqrt{\rho} t) .
$$

With these we can build the solutions of the Lienard equation that are

$$
x_{1}=\exp ((a+b) t) \quad x_{2}=\exp ((a+b+\sqrt{2 a b-c}) t) .
$$

For these we have the following cases:

Case 1. If $a, b, c \in \mathbf{C}$ and $(a+b+\sqrt{2 a b-c})=\alpha+i \beta=z$, then $x_{1}=\exp (\alpha t) \cdot \exp (i \beta t)$

1.1 If $\alpha \geq 0$ and $\beta=0$ then $x_{1}$ is not bounded in $-\infty$ and not bounded in $+\infty$

1.2 If $\alpha<0$ and $\beta=0$ then $x_{1}$ is not bounded in $+\infty$ and is not bounded in $-\infty$

1.3 If $\alpha=0$ and $\beta \neq 0$ then the solution $x_{1}$ is periodic. 
Case 2. If $a, b, c \in \mathbf{R}$ then $x_{1}=\exp ((a+b) t) \cdot \exp (\sqrt{2 a b-c} t)$

2.1 If $c<2 a b$ then we will have two solutions $x_{1}$ and $x_{2}$.

2.2 If $c>2 a b$ then $(a+b)+\sqrt{2 a b c}=(a+b)+i \sqrt{c-2 a b}$, that is to say that we would be in case one with $\alpha=a+b, \beta=\sqrt{c-2 a b}$ and also if $a=-b \Rightarrow \alpha=0$ or $a<-b \Rightarrow \alpha<0$ and $a>-b \Rightarrow \alpha>0$.

Our next aim will be to calculate the Galois groups associated with the equation $\ddot{y}=\rho y$, taking as a field of constants $K=\mathbf{C}$. This study can be seen in [15]. We must consider two cases:

Case 1. If $\rho=0$ then we will have the equation $\ddot{y}=0$, whose space of solutions is generated by $y_{1}=1$ and $y_{2}=t$. The Picard-Vessiot extension is $L=\mathbf{C}<t>$, where $\left\langle t>=\left\{\hat{k}_{0}+\hat{k}_{1} t: \hat{k}_{0}, \hat{k}_{1} \in \mathbf{C}\right\}\right.$. If we take the automorphism $\sigma \in D G a l<L / K>$ we check what form we should have.

First we must consider that $\sigma: L \rightarrow L$ so that $\left.\sigma\right|_{t}=$ identity, then $\sigma\left(y_{1}\right)=1=y_{1}$. Now

$$
\sigma\left(\partial_{t} t\right)=\partial_{t}(\sigma(t)) \sigma(1)=1=\partial_{t}(\sigma(t))
$$

Solving this equation by separable variables we have $\sigma(t)=t+C$. Therefore, the differential Galois group will be

$$
D G a l<L / K>=\left(\left\{\sigma_{c}: \sigma_{c}(y)=y+c \quad c \in \mathbf{C}\right\}, o\right)=\mathbb{G}_{a} .
$$

We can also see that:

$$
\sigma\left(\begin{array}{l}
y_{1} \\
y_{2}
\end{array}\right)=\left(\begin{array}{ll}
1 & 0 \\
c_{1} & 1
\end{array}\right)
$$

That is the differential Galois group is isomorphic to the group:

$$
\left.\left\{\left(\begin{array}{rr}
1 & 0 \\
c_{1} & 1
\end{array}\right): c \in \mathbf{C}\right\}, \bullet\right\}
$$

Case 2. If $\rho \neq 0$ we will then have the equation $\ddot{(y)}=\rho y$, taking the same field of constants from the previous case $K$. The base solutions for the equation are $y_{1}=\exp ^{\sqrt{\rho} t}$ and $y_{2}=\exp (-\sqrt{\rho} t)=\frac{1}{y_{1}}$. The Picard-Vessiot extension will be $L=\mathbf{C}<\exp (\sqrt{\rho} t)>$.

Now suppose that $\sigma \in D G a l<L / K>$ and let's see what action it performs on the solutions $y_{1}, y_{2}$.

$$
\sigma\left(y_{1}\right)=\sigma(\exp (\sqrt{\rho} t)) \Leftrightarrow \partial_{t} \sigma\left(y_{1}\right)=\sigma\left(\partial_{t} \exp (\sqrt{\rho} t)\right)=\sqrt{\rho}\left(y_{1}\right)
$$

Solving the differential equation: $\partial_{t} \sigma\left(y_{1}\right)=\sqrt{\rho}\left(\sigma\left(y_{1}\right)\right)$, by the method of separation of variables we have $\sigma\left(y_{1}\right)=y_{1}$.c. also as $y_{2}=1 / y_{1}$, then $\sigma\left(y_{2}\right)=\frac{1}{\sigma\left(y_{1}\right)}=\frac{1}{c . y_{1}}=\frac{1}{c} y_{2}$.

The Galois differential group will be

$$
D G a l<L / K>=\left(\left\{\sigma_{c} / \sigma_{c}(y)=c y \quad c \in \mathbf{C}\right\}, o\right)=\mathbb{G}_{m} .
$$

We can also see in this case that:

$$
\sigma\left(\begin{array}{l}
y_{1} \\
y_{2}
\end{array}\right)=\left(\begin{array}{cc}
c & 0 \\
0 & 1 / c
\end{array}\right)
$$

That is the Galois differential group is isomorphic to the group:

$$
\left\{\left(\begin{array}{cc}
c & 0 \\
0 & 1 / c
\end{array}\right): c \in \mathbf{C}, \bullet\right\}
$$

\section{Darboux theory of integrability}

Proposition 6.1. Consider the values of $\rho$ according to the table 1. The following statements hold: 
i. $\quad$ The invariant algebraic curves are $f_{1}=-v+\sqrt{\rho}$ y $f_{2}=-v-\sqrt{\rho}$ and their respective generalized cofactors $K_{1}=-1(v+\sqrt{\rho})$ y $K_{2}=-1(v-\sqrt{\rho})$.

ii. $\quad$ The Generalized Exponential factors are $F_{1}(v, x)=\exp (\sqrt{\rho} x+c)$

and $F_{2}(v, x)=\exp (-\sqrt{\rho} x+c)$. Moreover, their respective generalized cofactors are $L_{1}=(v, x)=\sqrt{\rho}$ y $L_{2}=-\sqrt{\rho}$

iii. The integrating factors are $R_{1}(v, x)=\frac{\exp (-2 \sqrt{\rho} x)}{(-v+\sqrt{\rho})^{2}}$ and $R_{2}(v, x)=\frac{\exp (2 \sqrt{\rho} x)}{(-v-\sqrt{\rho})^{2}}$

iv. The first integrals are given by

$$
\begin{gathered}
I_{1}(v, x)=\frac{-v-\sqrt{\rho}-\frac{\exp (-2 \sqrt{\rho} x)}{-2 \sqrt{\rho} \exp (-2 \sqrt{\rho} x+C)}}{-v-\sqrt{\rho}}(-2 \sqrt{\rho} \exp (-2 \sqrt{\rho} x)) \exp (1 / 4 \rho) \\
I_{2}(v, x)=\frac{-v+\sqrt{\rho}-\frac{\exp (2 \sqrt{\rho} x)}{2 \sqrt{\rho} \exp (2 \sqrt{\rho} x)+C}}{-v+\sqrt{\rho}}(2 \sqrt{\rho} \exp (2 \sqrt{\rho} x)) \exp (1 / 4 \rho)
\end{gathered}
$$

Proof. If we change the variable $v=\frac{\dot{y}}{y}$ about the equation $\ddot{y}=\rho y$ we will have the Riccati equation:

$$
\dot{v}=\rho-v^{2} \text {, }
$$

with solutions corresponding to $y_{1}, y_{2}$, respectively,

$$
v_{1}=\sqrt{\rho}, \quad v_{2}=-\sqrt{\rho} .
$$

Taking this equation as a foliation, the associated system will be:

$$
\begin{aligned}
\dot{x} & =1 \\
\dot{v} & =\rho-v^{2}
\end{aligned}
$$

and the associated vector field is $X=\partial_{x}+\left(\rho-v^{2}\right) \partial_{v}$.

Applying Lemma 1 of [3], we identify each of the Darboux integrability elements (also defined in Section 1.2), with $v_{\lambda}(x)$ as the solution:

a. Invariant Algebraic Curves:

$\left(f_{\lambda}(v, x)=-v+v_{\lambda}(x)\right)$

$f_{1}=-v+\sqrt{\rho} \quad$ and

$$
f_{2}=-v-\sqrt{\rho}
$$

Generalized Cofactors:

$\left(K_{\lambda}(v, x)=-N(x)\left(v+v_{\lambda}(x)\right)\right)$ in this case:

$$
K_{1}=-1(v+\sqrt{\rho}) \quad \text { and } \quad k_{2}=-1(v-\sqrt{\rho})
$$

b. Generalized Exponential Factor:

$\left(F_{\lambda}(v, x)=\exp ^{\int\left(\frac{N^{\prime}(x)}{2 N(n)}+v_{\lambda}(x)\right) d x}\right)$ in this case:

$F_{1}(v, x)=\exp \left(\int\left(\frac{0}{2}+v_{1}(x)\right) d x\right)=\exp \left(\int \sqrt{\rho} d x\right)=\exp (\sqrt{\rho} x+c)$

also

$F_{2}(v, x)=\exp \left(\int\left(\frac{0}{2}+v_{2}(x)\right) d x\right)=\exp \left(\int-\sqrt{\rho} d x\right)=\exp (-\sqrt{\rho} x+c)$

Generalized Cofactor:

$L_{\lambda}(v, x)=N^{\prime}(x) / 2+N(x) v_{\lambda}(x)$, in this case:

$L_{1}=(v, x)=N^{\prime} / 2+N v_{1}=\sqrt{\rho}, \quad L_{2}=(v, x)=N^{\prime} / 2+N v_{2}=-\sqrt{\rho}$

c. Integrating Factor:

$R_{\lambda}(v, x)=\frac{\exp \left(\int\left(-N^{\prime} / N-2 v_{\lambda}\right) d x\right)}{-v+v_{\lambda}}$ in this case:

$$
\begin{aligned}
& R_{1}(v, x)=\frac{\exp \left(\int\left(-1^{\prime} / 1-2 v_{1}\right) d x\right)}{-v+v_{1}}=\frac{\exp (-2 \sqrt{\rho} x)}{(-v+\sqrt{\rho})^{2}}, \\
& R_{2}(v, x)=\frac{\exp \left(\int\left(-1^{\prime} / 1-2 v_{2}\right) d x\right)}{-v+v_{2}}=\frac{\exp (2 \sqrt{\rho} x)}{(-v-\sqrt{\rho})^{2}} .
\end{aligned}
$$


d. First Integral For this case we must first calculate two elements:

1. $v_{(\lambda, 1)}=\left(\ln \left(y_{\lambda}\right)\right)^{\prime}$, in our case

$$
v_{(1,1)}=\sqrt{\rho} \quad \text { and } \quad v_{(2,1)}=\sqrt{\rho} \text {. }
$$

2. $v_{(\lambda, 2)}=v_{(\lambda, 1)}+\frac{\exp \left(-2 \int v_{(\lambda, 1)} d x\right)}{\int\left(\exp \left(-2 \int v_{(\lambda, 1)} d x\right)\right) d x}$, In our case

$$
\begin{aligned}
& v_{(1,2)}=v_{(1,1)}+\frac{\exp ^{-2 \int v_{(1,1)} d x}}{\int\left(\exp \left(-2 \int v_{(1,1)} d x\right)\right) d x} \\
& =\sqrt{\rho}+\frac{\exp \left(-2 \int \sqrt{\rho} d x\right)}{\int\left(\exp \left(-2 \int \sqrt{\rho} d x\right)\right) d x} \\
& =\sqrt{\rho}-\frac{\exp (-2 \sqrt{\rho} x)}{-2 \sqrt{\rho} \exp (-2 \sqrt{\rho} x)+C} \\
& v_{(2,2)}=v_{(2,1)}+\frac{\exp \left(-2 \int v_{(2,1)} d x\right)}{\int\left(\exp \left(-2 \int v_{(2,1)} d x\right)\right) d x} \\
& =-\sqrt{\rho}+\frac{\exp \left(2 \int \sqrt{\rho} d x\right)}{\int\left(\exp \left(2 \int \sqrt{\rho} d x\right)\right) d x} \\
& =-\sqrt{\rho}+\frac{\exp (2 \sqrt{\rho} x)}{2 \sqrt{\rho} \exp (2 \sqrt{\rho} x)+C}
\end{aligned}
$$

Now the first integrals are given by:

$$
I_{\lambda}(v, x)=\frac{-v-v_{(\lambda, 2)}}{-v-v_{(\lambda, 1)}} \exp \left(\int\left(v_{(\lambda, 2)}-v_{(\lambda, 1)}\right) d x\right) .
$$

Then:

$$
\begin{aligned}
& I_{1}(v, x)= \\
& \frac{-v-\sqrt{\rho}-\frac{\exp (-2 \sqrt{\rho} x)}{-2 \sqrt{\rho} \exp (-2 \sqrt{\rho} x)+C}}{-v-\sqrt{\rho}} \exp \left(\int\left(\sqrt{\rho}-\frac{\exp (-2 \sqrt{\rho} x)}{-2 \sqrt{\rho} \exp (-2 \sqrt{\rho} x)+C}-\sqrt{\rho}\right) d x\right) \\
& =\frac{-v-\sqrt{\rho}-\frac{\exp (-2 \sqrt{\rho} x)}{-2 \sqrt{\rho} \exp (-2 \sqrt{\rho} x)+C}}{-v-\sqrt{\rho}}(-2 \sqrt{\rho} \exp (-2 \sqrt{\rho} x)) \exp (1 / 4 \rho) \\
& I_{2}(v, x)= \\
& -v+\sqrt{\rho}-\frac{\exp (2 \sqrt{\rho} x)}{2 \sqrt{\rho} \exp (2 \sqrt{\rho} x)+C} \exp \left(\int\left(-\sqrt{\rho}+\frac{\exp (2 \sqrt{\rho} x)}{2 \sqrt{\rho} \exp (2 \sqrt{\rho} x)+C}+\sqrt{\rho}\right) d x\right) \\
& =\frac{-v+\sqrt{\rho}-\frac{\exp (2 \sqrt{\rho} x)}{2 \sqrt{\rho} \exp (2 \sqrt{\rho} x)+C}}{-v+\sqrt{\rho}}(2 \sqrt{\rho} \exp (2 \sqrt{\rho} x)) \exp (1 / 4 \rho)
\end{aligned}
$$

\section{Final remarks}

In this paper we studied one parametric family of linear differential systems from algebraic and qualitative point of view. Such parametric family comes from the correction of Exercise 11 in [2, §1.3.3], which we called Polyanin-Zaitsev vector field, see [1].

In this case we have taken a particular member of a family of equations, which were studied in general case in [1]. We have found critical points and the description near to these points. To our knowledge, we have obtained a new type of bifurcation. In the algebraic approach, the explicit first integral has been found using the Darboux method. Moreover, the differential Galois groups associated to solutions have also been found. 


\section{References}

[1] Acosta-Humánez P.B., Reyes-Linero A., Rodríguez-Contreras J., Algebraic and qualitative remarks about the family yy $y^{\prime}=$ $\left(\alpha x^{m+k-1}+\beta x^{m-k-1}\right) y+\gamma x^{2 m-2 k-1}$, preprint 2014. Available at arXiv:1807.03551.

[2] Polyanin A.D., Zaitsev V.F., Handbook of exact solutions for ordinary differential equations, Second Edition, 2003, Chapman and Hall, Boca Raton.

[3] Acosta-Humánez P.B., Pantazi Ch., Darboux Integrals for Schrodinger Planar Vector Fields via Darboux Transformations SIGMA, 2012, 8, 043.

[4] Van der Pol B., Van der Mark J., Frequency demultiplication, Nature, 1927, 120, 363-364.

[5] Nagumo J., Arimoto S., Yoshizawa S. An active pulse transmission line simulating nerve axon, Proc. IRE, 1962, 50, 20612070.

[6] Guckenheimer J., Hoffman K., Weckesser W., The forced Van der Pol equation I: The slow flow and its bifurcations, SIAM J. Applied Dynamical Systems, 2003, 2, 1-35.

[7] Kapitaniak T., Chaos for Engineers: Theory, Applications and Control, 1998, Springer, Berlin, Germany.

[8] Acosta-Humánez P.B., Lázaro J.T., Morales-Ruiz J.J., Pantazi Ch., On the integrability of polynomial fields in the plane by means of Picard-Vessiot theory. Discrete and Continuous Dynamical Systems, 2015, 35, 1767-1800

[9] Perko L., Differential equations and Dynamical systems, Third Edition, 2001, Springer-Verlag New York, Inc.

[10] Guckenheimer J., Nonlinear Oscillations, Dynamical Systems and Bifurcations of Vector Fields, 1983, Springer-Verlag New York.

[11] Nemytskii V.V., Stepanov V.V., Qualitative Theory of Differential Equations, 1960, Princeton University Press, Princeton.

[12] Christopher C., Llibre J., Integrability via invariant algebraic curves for planar polynomial differential systems, Annals of Differential Equations, 2000, 14, 5-19.

[13] Pantazi Ch., Inverse problems of the Darboux Theory of integrability for planar polynomial differential systems, PhD, 2004.

[14] Acosta-Humánez P.B., Teoría de Morales-Ramis y el algoritmo de Kovacic, Lecturas Matemáticas. Volumen Especial, 2006, 21-56.

[15] Acosta-Humánez P.B., Galoisian Approach to Supersymmetric Quantum Mechanics. PHD Thesis, Barcelona, 2009. Available at arXiv:0906.3532

[16] Acosta-Humánez P., Morales-Ruiz J., Weil J.-A., Galoisian Approach to integrability of Schrödinger Equation. Reports on Mathematical Physics, 2011, 67, 305-374.

[17] Acosta-Humánez P.B., Perez J., Teoría de Galois diferencial: una aproximación Matemáticas: Enseñanza Universitaria, 15, 2007, 91-102.

[18] Acosta-Humánez P.B., Perez J., Una introducción a la teoría de Galois diferencial. Boletín de Matemáticas Nueva Serie, 2004, 11, 138-149.

[19] van der Put M., Singer M., Galois Theory in Linear Differential Equations, 2003, Springer-Verlag New York.

[20] Morales-Ruiz J., Differential Galois Theory and Non-Integrability of Hamiltonian Systems, 1999, Birkhäuser, Basel.

[21] Lang S., Linear Algebra, Undergraduate Text in Mathematics, 2010, Springer, Third edition.

[22] Weil J.A., Constant et polynómes de Darboux en algèbre différentielle: applications aux systemes différentiels linéaires. Doctoral thesis, 1995.

[23] Pantazi Ch., El metodo de Darboux, en notas del primer seminario de Integrabiliad. Universidad Politecnica de Catalunya, 2005. Available at $h$ ttps : //upcommons.upc.edu/e - prints/bitstream/2117/2233/1/noinupc.pdf.

[24] Giacomini H., Gine J., Grau M., Integrability of planar polynomial differential systems through linear differential equations. Birkhauser, Rocky Mountain J. Math., 2006, 36, 457-485. 